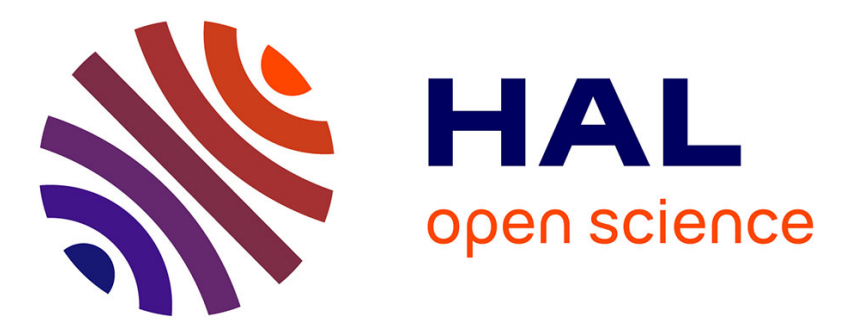

\title{
De retour de mon bain de tambours". Chants de transe du rituel maro chez les Toraja Sa'dan de l'île de Sulawesi (Indonésie)
}

Dana Rappoport

\section{- To cite this version:}

Dana Rappoport. De retour de mon bain de tambours". Chants de transe du rituel maro chez les Toraja Sa'dan de l'île de Sulawesi (Indonésie). Cahiers de Musiques Traditionnelles, 2006, 19, pp.93116. hal-00476153

\author{
HAL Id: hal-00476153 \\ https://hal.science/hal-00476153
}

Submitted on 14 Sep 2010

HAL is a multi-disciplinary open access archive for the deposit and dissemination of scientific research documents, whether they are published or not. The documents may come from teaching and research institutions in France or abroad, or from public or private research centers.
L'archive ouverte pluridisciplinaire HAL, est destinée au dépôt et à la diffusion de documents scientifiques de niveau recherche, publiés ou non, émanant des établissements d'enseignement et de recherche français ou étrangers, des laboratoires publics ou privés. 


\title{
"De retour de mon bain de tambours" Chants de transe du rituel maro chez les Toraja Sa'dan de l'île de Sulawesi (Indonésie)
}

\author{
DANA RAPPOPORT
}

En Indonésie, les faits de transe et de possession restent assez répandus; ils connaissent même un certain regain avec l'intrusion de religions importées. Ils ont notamment été étudiés sur les îles de Bali et Java (Zoete \& Spies 1938, Belo 1960, Jensen \& Suryani 1993), et, sur un plan musical, à Sumatra (Simons 1987, 1991), à Java (Kartomi 1973) et localement à Sulawesi dans deux populations, chez les Bugis (Hamonic 1987, Becker 2000) et les Wana (Atkinson 1989). Aucune étude générale sur ces "états altérés de conscience" (altered state of consciousness) n'a été entreprise sur un plan comparatif pour l'Indonésie entière.

En ethnomusicologie, après le travail théorique de Gilbert Rouget (1980), la transe a surtout été abordée par l'étude du lien entre musique et guérison (Roseman 1991, Friedson 1996, World of Music 1997, Gouk 2000) ou entre musique et esprits (Yamada 1997, Becker 2000). Depuis une vingtaine d'années, les découvertes des neurosciences ouvrent de nouvelles voies dans l'approche de ce phénomène (Becker 2004).

Dans la religion des Toraja de l'île de Sulawesi (anciennement appelée Célèbes) en Indonésie, religion qu'il conviendrait de nommer animiste (Tsintjilonis 2004), les divinités étaient, il y a encore peu de temps, régulièrement nourries et appelées par les humains ${ }^{1}$. Pour les attirer sur terre, ces derniers leur offraient les meilleurs morceaux de viande et les plus belles musiques. Jusqu'à la fin du $X X^{e}$ siècle, il fut encore possible d'observer les rituels dans lesquels les divinités étaient convoquées par le biais de transe et de chants (dans les rituels bugi' et maro) ou par celui d'offrandes instrumentales (lors du rituel d'éviction de la variole, ma'pakorong). 
Je présente ici les chants d'un rituel majeur, devenu très rare aujourd'hui en raison de son interdiction par les congrégations chrétiennes dès les années $1930^{2}$. Vulgairement appelé maro, "fou", ce rituel est exécuté après la moisson, d'octobre à février, avant de recommencer un cycle agraire. Cette période est consacrée aux obligations rituelles relatives aux terres pendant qu'elles sont au repos (Coville 1989: 108) ${ }^{3}$. Si les grains poussent vite, alors la famille peut décider de faire un rituel qui doit purifier le village et favoriser la fertilité du riz. Selon l'officiant - "celui qui sait", to minaa - Ne'Ambaa (c.p. 1993), les trois raisons d'exécuter un rituel maro sont de "fertiliser les terres" (umpopembura padang), de "guérir les gens de la variole" (yake denni tau ma'bulan) et de "guérir une personne de folie" (yake denni tau bombo-bomboan). Dans certains endroits, le maro est aussi exécuté pour la conversion de l'âme d'un ou plusieurs défunt(s) vers le soleil levant (maro baté ma'pabalik).

Dans ses variations, le rituel fut déjà observé par plusieurs ethnologues (Zerner 1981; Nooy-Palm 1986, Volkman 1985, Coville 1988, 1989). Avant la Seconde Guerre mondiale, le linguiste $\mathrm{H}$. van der Veen (1979) recueillit quelques extraits de chants qu'il publia en néerlandais. Mes propres sources sonores et écrites furent enregistrées lors d'un rituel maro de six jours et six nuits. Celui-ci combinait deux buts: purifier le village et retourner l'âme des défunts. Je revins de ce rituel avec 34 heures de son enregistré à partir desquelles je fis transcrire 15000 vers issus des chants exécutés à l'intérieur et à l'extérieur des maisons. Tous les chants de ce rituel se nomment gelong maro ("chant fou"). Sur ces 15000 vers, 500 seulement furent traduits et étudiés: ceux qui étaient chantés pendant les transes à l'extérieur ${ }^{5}$. D'autre part, j'ai recueilli hors contexte auprès d'un officiant, la matrice ordonnée de ces chants, le "chant de l'être-en-divinité" (gelong kandeatan), composé de 230 vers. On peut se demander comment il est possible de fonder un savoir sur un si faible échantillon verbal. En fait, les chants de transe étant très répétitifs, ils sont représentatifs de ce qui a lieu dans une séance de transe. C'est par l'analyse de ces vers et des motifs musicaux, ainsi que par l'observation des gestes que j'ai pu entrevoir l'organisation des transes du rituel maro.

2 Les données présentées ici ont été recueillies au cours de deux rituels maro auxquels j'ai assisté dans la même région, lors d'un séjour d'un an en pays toraja en 1993.

3 Le gouvernement voudrait aujourd'hui que les villageois entament une nouvelle plantation immédiatement après la récolte, ce qui représente une violation de la règle puisque, dans le calendrier agraire, cette période doit être consacrée aux rituels.

4 Le terme gelong ou kelong est le nom des chants de transe des rituels maro et bugi'.

5 Des enregistrements sont accessibles (Rappoport 1995). L'intégralité des chants sera audible dans un DVD-Rom (Rappoport: à paraître). 


\section{Le rituel des «fous" (ma'maro)}

\section{Journal}

Village de Torea, canton Sesean, région Toraja Sa'dan.

MERCREDI 17 NOVEMBRE 1993. Depuis trois jours, des officiants, invités de toute la région, sont venus en petits groupes, psalmodier nuit et jour. lls se répartissent en cinq groupes pour officier dans cinq maisons. Dans les pièces centrales, bondées, impossible d'étendre ses jambes. Luther, mon assistant, enregistre dans une maison depuis trois nuits l'officiant $\mathrm{Ne}$ ' Mendo; de mon côté, j'écoute dans une autre maison les psalmodies de Ne'Sampe, qui ne remplit pas sa tâche. La qualité des récitations est inégale: certains, paresseux, se contentent de dire les paroles à toute allure, hachent leur discours, s'endorment, puis se relèvent pour poursuivre - attitude dangereuse pour la suite, me dira-t-on. Dans d'autres maisons, des officiants, plus appliqués, récitent la totalité d'une parole dont le sens m'est totalement inconnu. En mauvaise voie, je rejoins Luther dans une autre maison. II n'a pas dormi. Déjà treize cassettes pleines. Grande difficulté à trouver quelqu'un qui puisse m'expliquer ce qui se passe.

Chaque matin, chaque soir, offrandes aux divinités et aux ancêtres. Avant de sacrifier, il faut chanter. Après, chanter encore. Dire et redire que les divinités ont bien mangé. Lentement d'abord, puis rapidement. C'est le "chant de cuisine" (gelong dapo') qui se répète non seulement chaque soir, mais qui en plus décline en série toutes les entités invisibles à qui ont été offerts les poulets:

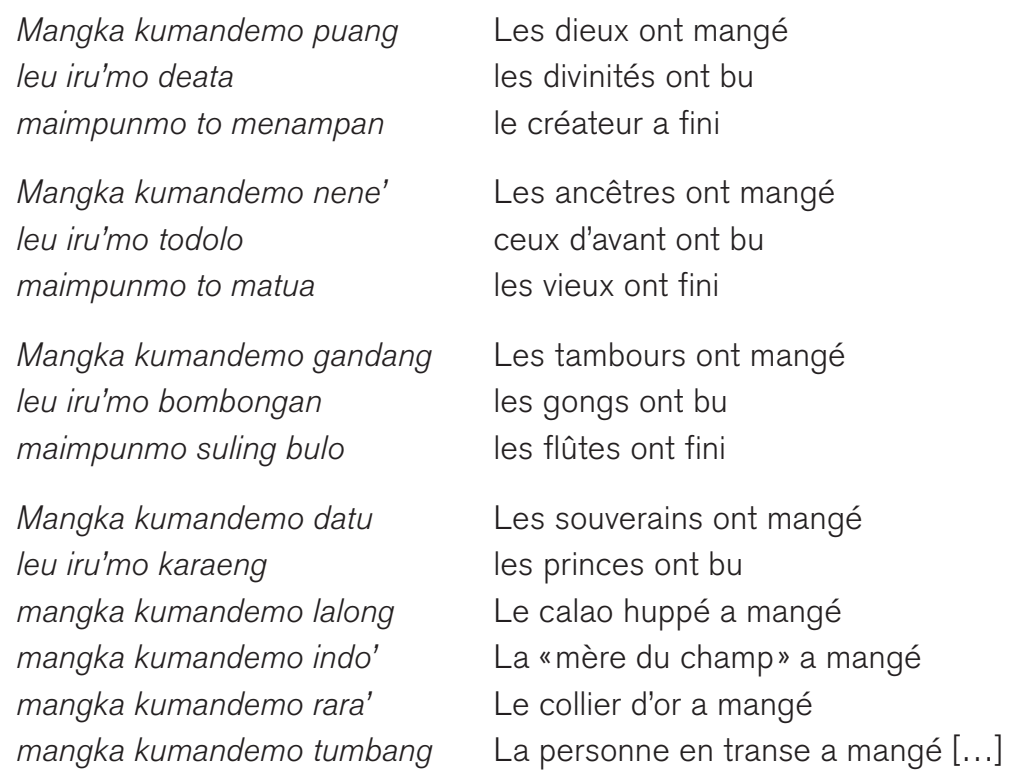

(Gelong dapo", "chant de cuisine», 18 novembre 1993) 
Chaque soir, poulets égorgés à n'en plus finir, recherchés dans tous les environs pour ce rituel aux centaines de poulets.

Jeudi 18 novembre. Des transes ont lieu dans la cour de chacune des maisons. Des hommes chantent en sautant. Déchaînées, cheveux déliés, certaines femmes tournent sur elles-mêmes puis partent à la renverse. Elles se flagellent. Peu après, un homme se dégage du groupe, se fouette avec les feuilles rouges de cordyline, boit de la boue, puis se mutile en incisant son front à l'aide d'un poignard. Le sang coule. Un chœur de femmes chante:

$\begin{array}{ll}\begin{array}{l}\text { Tasirere'-rere' lendong } \\ \text { tasi'pa'tallu masapi }\end{array} & \begin{array}{l}\text { Réciproquement hachons l'anguille } \\ \text { réciproquement tranchons par trois } \\ \text { le serpent d'eau }\end{array} \\ \begin{array}{l}\text { tasiimpa' bale rante } \\ \text { réciproquement coupons le poisson de terre } \\ \text { kumande lannako liu } \\ \text { ma'kasolang-solanganko }\end{array} & \begin{array}{l}\text { Fer, entre dedans } \\ \text { profondément tu te nourris } \\ \text { défais et détruis }\end{array} \\ \begin{array}{l}\text { Tikalulunmoko bassi } \\ \text { tilu'pi'moko mataran } \\ \text { patondon patomaliko }\end{array} & \begin{array}{l}\text { Fer, amollis-toi } \\ \text { chose coupante, plie-toi } \\ \text { enroule-toi de tous côtés }\end{array}\end{array}$

(Gelong Kandeatan, "chant de l'être-en-divinité", vers 46-54)

Les femmes poursuivent leur chant autour de l'homme scarifié qui saigne. II cherche quelqu'un qui veuille guérir par le sang. II porte un enfant, touche des adultes. Au cours du chant, le sang, en train de perler, s'arrête immédiatement. Une femme en transe demande un tambour, fait trois fois le tour du tambour et monte dessus. Le chœur de femmes chante:

Daomo' tangkena gandang mengurapakna tandilo ma'tondon penainna

Tondok boro toda dao banua mapia toda boro pangrantean toda

Kari' la sa'timo dao kari' tang la sule lemmo tang la balik bulo lemmo

Napopedampi to tumbang napotamba' to malangi' burra to kandeatan
Je suis montée sur le tambour en haut de la cithare sur la pointe du sabre Assurément, là-haut, un très beau pays des maisons vraiment belles assurément, là-haut, une belle contrée Je serais presque restée là haut j'étais sur le point de ne pas rentrer je ne serais pas revenue II guérit la personne en transe il soigne le monte-au-ciel crachat des êtres en divinité 
Nasule kale datunna

to kuli' ampu lembangna

urruru tampa dolona

sola tasim bulayanna

Anna burinti tumende

anna langkan boro tia'
Afin qu'ils retrouvent un corps de roi

avec une peau saine

retrouvent la forme du début

avec une force rayonnante

Et ils courent tel l'échassier

et ils volent tel le rapace

Quand la personne descend du tambour, le chœur chante:

Sulemo' mendio' gandang membollo-bollo tandilo

melangi'ri suling bulo

Ta'pa passakkemo gandang

bua uranmo tandilo

Napopedampi to tumbang

napotamba' to malangi'

burra to kandeatan
De retour de mon bain de tambours rincée aux cithares lavée aux flûtes

Le tambour est retombé, telle une bénédiction la cithare goutte tels des fruits d'eau

II guérit la personne en transe

il soigne le monte-au-ciel

crachat des personnes "en divinité»

(gelong kandeatan, "chant de l'être-en-divinité», 108-133)

Je commence à m'habituer sans bien comprendre. Souvent, je désespère d'être perdue dans cette suite de paroles et d'actes apparemment sans queue ni tête. J'enregistre des chants qui semblent discontinus à l'extérieur des maisons et, inversement, continus à l'intérieur. Les données s'amassent. J'enregistre tout ce que je peux, tout ce que je vois, tout ce que j'entends. J'enregistre dehors, Luther dedans.

VENDREDI, 6H DU MATIN. On se lève en retard, Lumbaa a déjà recommencé à psalmodier dès l'aube. Journée d'offrandes de poulets aux officiants. "Faire manger", ici, signifie "offrir". Dans tous les rituels, il faut nourrir: nourrir les officiants (ma'pakande to minaa), nourrir les morts dans les funérailles (ma'pakande nene'). Ce qui est offert n'est pourtant pas mangé: la viande passe à la trappe, les chiens la mangent. Des centaines de foies de poulets sont retirés des corps. Les poulets, fumés, sont alors placés dans des corbeilles dans la cour. De grands mâts tridents chargés de poignards, de tissus cérémoniels et de plante cordyline sont préparés.

SAMEDI. Dernier jour, grand jour, celui de "l'érection des mâts cérémoniels" (ma'baté). L'agitation est à son comble. La mère chez qui je loge se lève dès $3 \mathrm{~h}$. du matin pour préparer la nourriture. De chaque maison sortent les tambourinaires et les danseuses, les officiants. Tous sortent en chantant pour accompagner les très hauts mâts en bambou, à trois branches. Cinq fois cinq: soit cinq groupes de tambourinaires, cinq groupes d'officiants, cinq mâts, cinq groupes de danseuses. 
Tous se rejoignent sur le grand champ en dehors du village, situé vers le soleil levant. Les mâts sont plantés dans une euphorie indescriptible, puis chantés; les hommes sautent frénétiquement, les femmes dansent, partent à la renverse, certains se scarifient. Et dans une joyeuse polymusique, les divinités sont conviées une dernière fois à descendre pendant que les hommes chantent:

Anna deatai tondok anna puang di pangleon datu lan sa'de banua

Maripiko kuondoi rapa'ko kutarandakki kupembulisu-lisui

Penduan ponnomo tondok pentallun kapalenanmo ra'dak tandung sea-sea

La lao rokkomo mai laomo sambalin mai bu'tu lamban diong mai

Pealla'ko padang-padang padang-padang tang kulese

Lakkia' kipelalanni botto kidakaran embe'

Sape-sape to Balanda sokko' to mappau-pau inde to kandeatan

Tiro-tirooi lako tungka para'pai mata

Borrong tongan dukku tongan tipamian-mian tongan

Tiumba'mo sape-sape dollokmo kundae pangka kundae pangka to Bone

Sape-sape to Balanda sumonglo'mo to Sesean dollokmo to Lindo Tau
Et les divinités de ce village et les dieux de ce hameau déités à côté de la maison ${ }^{6}$

Reste calme je vais piétiner tiens-toi tranquille je vais fouler la terre je vais tourbillonner

Deux fois le village a été rempli trois fois il a débordé le grand champ entièrement comble

Vont descendre ici descendre de l'autre côté sont en train de traverser d'en bas Avancent entre les ixores écarlates ixores écarlates ne piétine pas

Les plantes épineuses nous escaladons sur les plantes violettes nous glissons

Parures des Balanda ${ }^{7}$ couvre-chefs des gens casqués voici les êtres-en-divinité

Regarde là-bas je vois vraiment rouge

Vraiment rougeoie, vraiment brille vraiment tel l'éclair

Surgissent les parures arrivent les tissus extraordinaires sarong merveilleux de gens de Boné

Cueillette des Balanda ceux de Sesean sont descendus ceux de Visage Humain sont arrivés

6 Demande de permission aux divinités locales afin qu'elles acceptent les divinités étrangères qui vont descendre dans le corps des gens en transe.

7 Balanda: «Hollandais, étranger», cf. infra, p. 107. 


$\begin{array}{ll}\begin{array}{l}\text { Anna deata di Limbong } \\ \text { anna puang dipangleon } \\ \text { datu disa'de banua }\end{array} & \begin{array}{l}\text { Et les divinités de Limbong } \\ \text { les dieux de ce village } \\ \text { déités à côté de la maison }\end{array} \\ \begin{array}{l}\text { Tang mondo-mondo disa'bu' } \\ \text { tang maundan dipokada }\end{array} & \text { Ils n'en finissent pas d'être nommés } \\ \text { tang leluk dipau-pau } & \text { ils n'en finissent pas d'être dits } \\ \text { La kupokada rara' ko } & \text { leur évocation n'est pas modifiée } \\ \text { la kusa'bu' bulayanko } & \text { Je parlerai de ton joyau } \\ \text { kugente' kandaureko } & \text { je citerai ton or }\end{array}$

Extraits de chant gelong maro, collectés en novembre 1993.

J'ai longtemps cherché le sens de ces actes, de cette dramaturgie unique. Et ce n'est que bien plus tard que je l'ai entrevu, grâce à l'étude des chants. Tout comme dans les funérailles, la parole chantée commente l'action rituelle. Avant d'examiner ces chants, présentons les différentes séquences d'une manière plus détaillée:

\section{Les séquences du rituel}

A Torea, cinq maisons, représentant cinq ramages ${ }^{8}$, ont décidé d'organiser un rituel maro pour purifier le village et faire remonter l'âme de certains défunts au Levant. Les offrandes sont offertes chaque jour aux divinités, en bas pour Pong Tulak Padang, en aval (sau' lalanna sukaran aluk), en amont (rekke lalanna bulan tasak), vers les ancêtres au soleil Couchant et en haut pour un des vieux dieux. Les six jours de rituel, du 15 au 20 novembre 1993, se sont déroulés ainsi.

\section{Premier jour. Rites de séparation}

Plusieurs rites sont exécutés pour la bénédiction du village (sambe’tondok, "faire des prières aux divinités par l'offrande de poulets"), les offrandes ouvrent la période d'interdits: interdit de manger des piments et du porc, interdit de travailler... Les récitations gelong, psalmodiées à l'intérieur des cinq maisons par cinq groupes d'officiants, commencent.

\section{Deuxième jour. "Aller sur le marché» (ma'pasa')}

Le champ cérémoniel (pasa', "marché») est délimité par des offrandes (piong). Dans les cours des maisons, des offrandes aux ancêtres et aux divinités sont faites sur un tambour. Le tambour est "ensanglanté par trois sangs" (ditallung

8 "Ramage» correspond au terme toraja rapuan. Il désigne un groupe de filiation cognatique se réclamant d'un ancêtre commun, fondateur de la maison tongkonan; il désigne en fait une structure très vaste représentée lors des grands rituels (Nooy-Palm 1979: 26). 
rara'i). II représente un attribut du ramage: chaque tambour participant au rite sera apporté le dernier jour sur le grand champ.

\section{Troisième jour. Procession vers la pierre de Landorundun}

Les femmes stériles se rendent à la pierre de Landorundun pour favoriser leur fécondité. Personnage mythique, fille de l'union de Lambe' Susu et de Salokan, Landorundun aux longs cheveux est considérée comme la plus belle femme de la région de Sesean. Elle avait l'habitude de se baigner à une source dont la trace est encore visible aujourd'hui par la présence d'une large pierre. Un de ses cheveux s'est perdu et a été retrouvé par le Prince de Boné qui l'a demandée en mariage. Le soir, dans les cinq maisons, est psalmodié le gelong Boné ("chant de Boné») relatant ces faits.

\section{Quatrième jour. "Cracher, pulvériser" (ma'burra)}

Le rite ma' burra consiste à guérir ou à prévenir la maladie des enfants en les lavant et en leur administrant des pulvérisations buccales (ma'burra). Ce jour-là, dans chacune des maisons, ont lieu des séances de transe. Les femmes tournoient sur elles-mêmes, perdent conscience. Différentes scènes rituelles à caractère ludique ont alors lieu: elles marchent sur des braises, se cachent sous des tissus, s'envoient des paniers à la figure; à un certain moment, une des femmes en transe, contenue par plusieurs hommes, est ramenée à son état ordinaire par le jeu de deux petites flûtes horizontales en bambou. Une autre femme en transe monte sur le tambour puis retrouve progressivement ses esprits pendant que le chœur poursuit le chant. Un homme ("en divinité ", to kandeatan) se lacère le front, saigne, boit de la boue, se perce la langue et applique son sang sur un enfant. Pendant ce temps, un chœur mixte chante lentement.

\section{Cinquième jour. "Faire manger les officiants" (ma'pakande to minaa)}

La récitation gelong est énoncée sans interruption, dès le lever du jour et jusqu'au petit matin suivant. C'est le jour du rite ma'pakande to minaa ("faire manger les officiants"). Dans chaque maison, une grande agitation a lieu: plus de cent poulets sont tués, cuits sans être découpés puis partagés entre les officiants dans des corbeilles (rakki). L'après-midi ont à nouveau lieu des chants, des danses et des transes. Un homme en transe porte deux enfants à cheval sur ses épaules (geste à valeur prophylactique $)^{9}$. Le chant gelong ma'pakumpang commence le soir, la veille du grand jour.

9 Ces gestes sont évoqués dans le chant gelong: sae ma'sompo ma'kepak/ma'takia' patomali: «ils viennent en portant sur les épaules, en portant par dessous les bras». 


\section{Sixième et dernier jour. "Aller sur le marché" (ma'pasa')}

C'est le grand jour, le dernier jour. Chacune des cinq maisons érige son mât trident sur lequel sont empilées de précieuses draperies maa'. Vers midi, les mâts sont apportés sur le grand champ. Chaque maison apporte son mât en chantant à tuetête le refrain "cueillette des étrangers", Sape-sape to Balanda. Chaque départ, de la maison au champ cérémoniel, est joyeux, animé, débridé. Les hommes arrivent sur le champ cérémoniel en sautant et en criant, suivis par la grande famille, les officiants, les groupes de danseuses, les joueurs de tambour, tous portant les feuilles rouges de la cordyline. Après avoir fait trois fois le tour du champ, ils plantent chaque mât face au Levant. Le monde afflue. Les familles s'installent près de leurs mâts respectifs. Chaque maison a donc son mât cérémoniel baté, son groupe de danseuses gel/u', son ou ses officiants to minaa, son tambour gandang, ses alliés qui ont préparé des gâteaux de riz (katupa).

A nouveau des transes ont lieu, les divinités sont conviées à descendre sur le lieu cérémoniel; pendant ce temps, chaque mât est "chanté" par la psalmodie gelong baté. Devant chaque mât, un groupe de jeunes filles en habits cérémoniels, le front ceint d'un bandeau du fruit tarrung, exécute la danse gellu', au son du tambour frappé à l'aide de baguettes par quatre garçons. La danse est simple, calme et gracieuse, les filles sont en rangs parallèles. Chaque groupe de danseuses est constitué de filles de chacune des cinq maisons ${ }^{10}$.

C'est une apothéose visuelle: sur le lieu dégagé, dans un grand paysage ouvert surmontant la vallée, les mâts sont plantés, multicolores, grandioses, les danseuses sont éclatantes, les hommes s'agitent en tous sens. C'est une apothéose sonore: sur le même espace, tambours, chant saccadé maro, chant des officiants gelong bate, cris, chant des femmes en jaune gelong bainé, chant bugi'... La fête s'achève sur un combat rituel de pied sisemba très violent dans un champ à côté.

Comment comprendre ce que signifie ce rituel? A quoi servent les chants dans cette grande dramaturgie d'interactions?

10 En 1939, les danseuses chantaient en dansant, ce qui n'est plus le cas aujourd'hui; cependant, Claire Holt n'a pas assisté au rituel. 


\section{Le lexique}

Le lexique permet d'éclairer certains points. Ce rituel est nommé de trois manières: maro signifie «fou, dément» - etj'assiste bien à une succession de représentations démentes et d'actes fous (scarifications, flagellations, sauts débridés, chutes); en outre, à la fin du rituel, un combat de pied (sisemba) extrêmement brutal est autorisé, dernier moment d'une violence encadrée ${ }^{11}$. Le rituel se nomme aussi "cueillette de la cordyline" (sapean tabang): tout au long du rituel, les feuilles rouges de la plante cordyline servent à la fois de fouets de flagellation et de remède pour guérir. Enfin, ce rituel est encore nommé "mât(s) descendu(s) [du ciel]» (bate manurun). De grands mâts sont érigés le dernier jour, certains sont dressés en réponse aux funérailles comme les répliques du grand mât funéraire ${ }^{12}$; le rituel se nomme alors maro baté ma'pabalik ("rite des fous du mât retourné»), ou bate umpabalik bandera ("mât qui retourne le mât du défunt»). Autrement dit, la continuité entre les rituels du Couchant (rites de mort) et les rituels du Levant (rites de vie) se fait par l'érection de bambous de taille croissante et de mâts tridents qui sont chantés et qui se retrouvent dans la chaîne des différents rituels.

Les personnes en transe sont désignées dans les chants de trois manières. Le terme courant est formé sur la racine "divinité» (deata). La personne en transe "fait divinité" (to ma'deata) $)^{13}$, "est en divinité" (to kandeatan). Deux autres termes, toujours couplés, sont couramment utilisés: tumbang/malangi'. Tumbang ("danser et s'agiter en tous sens " ") et malangi' (langi', "ciel») sont ceux qui dansent et tournoient pendant qu'un chœur d'hommes chante. Souvent, ce sont des femmes ${ }^{15}$. Le chant répète:
Napopedampi to tumbang
Il guérit les "personnes en transe» (to tumbang)
napotamba' to malangi'
il soigne les "monte-au-ciel» (to malangi')
burra to kandeatan
crachat des personnes "en divinité» (to kandeatan) ${ }^{16}$

\footnotetext{
11 Le sisemba' est une lutte interdisant l'emploi du haut du corps. Deux garçons se tiennent par la main et s'élancent contre deux autres adversaires. L'un donne un coup de pied tandis que son partenaire joue le rôle de défenseur, en le retenant dans son équilibre. En raison de sa violence, ce jeu fut souvent interdit par le gouvernement.

12 Dans les plus grandes funérailles, un mât appelé bandera au nord ou bate lepong est érigé pour le défunt (Rappoport: à paraître)

13 De deata: "divinité", et du préfixe verbal ma' qui indique l'action. II existe une autre expression qui suggère que le sujet est passif: "celui qui est pris par sa divinité» (tonala deata'na).
}

14 Tumbang: "danser à la fête maro pour trouver le pouvoir magique sous le commandement des divinités" (in Veen \& Tammu 1972).

15 II est probable que tumbang désigne une femme. Trois éléments permettent de le penser: 1) à la fête bua' kasalle, le titre de tumbang est exclusivement réservé aux femmes; 2) lors du maro, ce sont surtout les femmes qui tournoient sur elles-mêmes et entrent dans un état second; 3) le personnage mythique du maro est la divinité féminine Indo' Belo Tumbang. D'après H. NooyPalm également (1986: 130), tumbang désignerait une personne de sexe féminin. Elle fonde sa position sur un vers de gelong maro reproduit par van der Veen (1979: 52-3, vers 43).

16 Burra: "cracher, pulvériser, asperger». 
La personne en transe est guérie, le possédé est soigné, la personne en divinité "crache». Qui sont véritablement ces personnes nommées ici dans les chants? Sont-elles distinctes?

\section{La guérison}

Le mythe explique la fonction du rite. L'histoire est racontée dans un passage du mythe La Passomba tedong ("purification du buffle") énoncé lors du rituel merok, avant le sacrifice du buffle (Veen 1965: 142, v. 726 à 743):

Le premier rituel maro eut lieu au ciel pour guérir l'enfant du «Vieux Dieu» Puang Matua. Datu Bainé "Reine Femme" (ou Banno bulaan), une enfant du grand dieu, tombe malade. Un messager est envoyé pour aller chercher la divinité - Indo'Be/o Tumbang, "Mère Ornement des gens en transe» - qui permettra de guérir l'enfant. Celle-ci se met en route avec ses remèdes qui seront pulvérisés ou crachés (burra) sur les malades [à la fête maro, les malades sont guéris par le "crachat" du guérisseur, doté d'un pouvoir surnaturel, éprouvé par le feu et par le fer]. Elle part au centre du ciel pour discuter avec le vieux Puang Matua, elle mâche la chique de bétel avec lui, puis dit: "nous voici avec le remède à cracher [ou pulvériser] mais notre intérieur est dérouté, le fond de notre intériorité est confus, c'est comme si nous allions dans une forêt impénétrable, comme si le chemin emprunté était épais et souterrain, ce n'est pas un chemin ouvert, ce n'est pas un chemin dégagé» (v. 733-734). Elle exige alors qu'on appelle Kambuno Langi [premier officiant to minaa au monde supérieur] afin qu'il fasse un rite expiatoire avant qu'elle ne guérisse elle-même le patient. II monte sur la maison de fer (banua bassi) et brise des nervures de feuilles de palmier pour compter le nombre de transgressions commises (rebongan didi), puis cueille des feuilles de la plante rouge cordyline (tabang) [sapean tabang, nom du rituel maro]. L'officiant fait l'offrande devant la maison de fer. Puis la divinité guérit l'enfant, en crachant des feuilles bien mâchées, rouges comme sang. Alors le grand mât est érigé et flotte dans le vent. Les humains, les animaux et les plantations sont protégés.

On note d'une part, l'importance de la femme dans la guérison - c'est une divinité féminine qui apporte le remède -, d'autre part, le rôle du sang évoqué par le biais de la plante rouge cordyline, la place de la pulvérisation buccale et enfin l'érection du grand mât (bate, bandera). Les paroles des chants ne cessent de le répéter: le sang, les feuilles rouges et la pulvérisation buccale serviront de remèdes pour retrouver un corps parfait. Le mythe inscrit le rite dans sa fonction curative et prophylactique. Etrangement, le mythe ne dit rien des transes pourtant largement présentes dans le rite. 


\section{La folie}

Santé et force de vie sont les deux biens recherchées par le biais de la folie mimée, manifestée par les transes et par les chants des hommes qui sautent de manière grotesque en chantant à tue-tête. Ces hommes, qui se comparent à des singes, sont appelés "ceux qui sautent" (to ma'panondo), ou "ceux qui font tomber [les divinités] ] ${ }^{17}$ (to ma'parondon). En proférant des rires outrés, ils s'excitent, se chauffent, se donnent de l'ardeur, puis entonnent le chant en bondissant sur place tels "des fous» (to maro). A ce moment, quelques femmes d'âge mûr dansent en sautant, cheveux déliés, sarong défait puis tournoient dans le sens inverse des aiguilles d'une montre. Elles exécutent des actes extraordinaires. Soudainement, elles accèdent à un état second, les yeux fermés, la tête révulsée en arrière. Un second chant, plus calme - le chant de la scarification (sampa-sampa to mantere) -, accompagne ensuite les incisions d'un officiant rituel.

Le son des flagellations, des tambours, des polymusiques en désordre, la couleur rouge sang omniprésente, le sang de l'homme scarifié: tous ces éléments font du maro un rite de l'outrance, de la folie, seul rite impliquant un déchaînement et un désordre des corps.

\section{Les chants de transe}

Les séances de transe ont eu lieu durant les trois derniers jours, d'une part dans les cours des maisons et, le dernier jour, sur le grand champ cérémoniel "marché», lieu d'échange entre humains et divinités. A chaque séance, deux types de chants peuvent être distingués. Rapide, le chœur masculin appelle les divinités à descendre; lent, le chœur féminin évoque les visions de la personne "en divinité". Tous deux diffèrent par les protagonistes, les actes accomplis, les motifs musicaux et les paroles.

\section{1. "Chant de l'arrivée des divinités" (gelong ma’pasae deata)}

Le premier chant porte plusieurs noms: nondo muane ("sauts des hommes"), gelong unnondo ("chant à sauter"), gelong ma'pasae deata ("chant de l'arrivée des divinités"). Lorsqu'un petit chœur d'hommes chante, quelques femmes dansent puis entrent en transe. Des hommes les retiennent, tentent de les contenir. Ces femmes font des actes extraordinaires, marchent sur des braises, sur des couteaux, elles "font divinité» (to ma'deata). Leur exigence doit être satisfaite. Si la personne en

17 De rondon, "tomber", à propos des feuilles des arbres. La chute est ici celle des divinités "tombant» sur terre. 
transe demande une épée, les Toraja disent qu'elle se tranchera la tête et se couvrira le visage de sang. Si c'est une lance, elle s'assiéra dessus. Si c'est un bambou, elle montera en haut et restera assise en jouant de la flûte. Si c'est une femme, elle ne grimpera pas au bambou, mais exigera une échelle de poignards et montera dessus sans se blesser (Wilcox 1949: 344). A la fin, après une heure environ de démence, elles sont ramenées à leur état de conscience normale grâce à leur demande exaucée, par des objets ou des sons.

L'agitation frénétique est déclenchée par le chant saccadé des hommes, mené dans une grande excitation, au son des cris, des tambours et des claquements de flagellations. Les divinités, une fois descendues dans le corps des femmes, incitent les personnes en transe à faire des actes hors du commun, ce qui constitue la preuve de leur présence.

Ci-contre, de haut en bas:

"Chant pour sauter" gelong unnondo.

Femme en transe sur le champ cérémoniel.

Torea, 1993.

Une femme en transe marche sur des braises, 1993.

La femme en transe tombe à la renverse, 1993.

Ci-dessous:

Un homme s'est ouvert le front à l'aide d'un couteau.
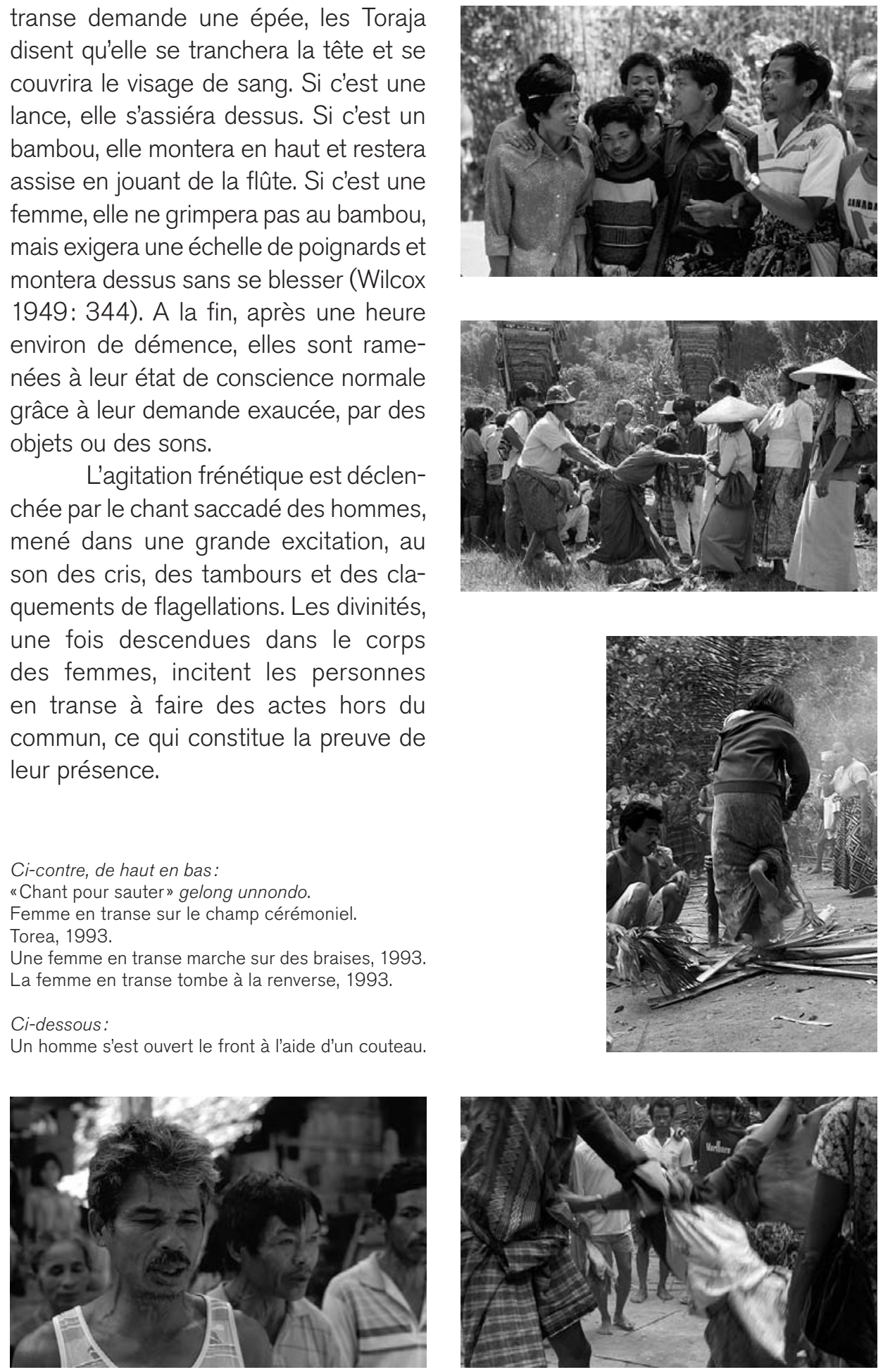
Sur le plan musical, c'est une scansion collective qui s'effectue sur deux ou trois phrases de huit temps dont la plus courante est construite sur un intervalle de tierce majeure, aux paroles intelligibles et au tempo rapide:

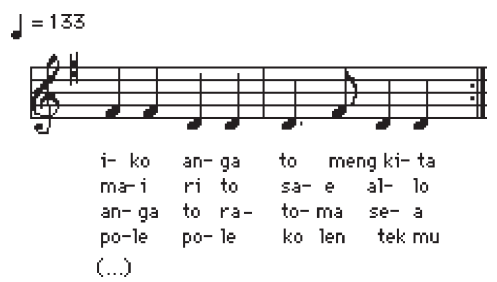

Motif de chant gelong unnondo, "chant à sauter"

Le motif musical ne se réfère pas à une divinité particulière; ici, les divinités sont indifférenciées ${ }^{18}$. Certaines fois, le meneur ("mère du chant», indo' gelong) embellit la profération en variant les motifs, en dynamisant davantage la formule:

A

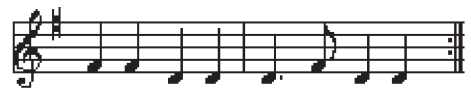

A1
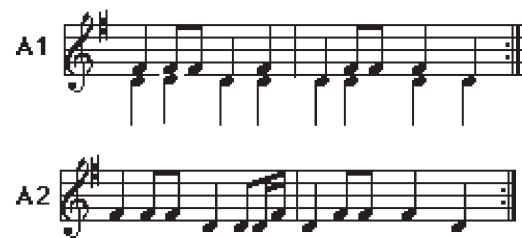

Cette profération ne faisant l'objet d'aucun développement musical, d'aucun changement de tempo, n'importe qui peut entrer dans le chant.

De plus, ce chant ne suit pas de continuité narrative; ses fragments sont égrenés en désordre. II évoque la situation présente, le public, le jour de fête, le lieu cérémoniel, les personnes qui dansent et tombent en transe; il annonce au public la descente des divinités sur terre:

Iko angga to mengkita

mairi' to sae allo

angga to ratu masiang

Poli'-poli'ko lentekmu

kalili'ko kambutu'mu

embongko tiku lilingmu
Toi public qui assiste tous les gens présents aujourd'hui tous ceux arrivés en ce jour

Change ton pied de place recule ton talon fais de la place autour de toi
18 Pendant ce chant sauté, les chanteurs peuvent intercaler librement le chant sauté nondo bugi'. S'ils chantent le bugi' (c'est-à-dire avec les paroles et la musique du bugi, un autre rituel de transe) alors c'est l'esprit bugi' qui vient, qui descend. Les mélodies des scansions du maro diffèrent de celle du bugi' mais plusieurs versets sont communs. 


\author{
Nasalembe' ako tumbang \\ natodoako malangi' \\ natekka-tekkaiako
}

Ammu kuamo kumua ammu patende ma'kada bangaran kupau-pau

Male mati' tang memanuk tang ussopatian langkan tang umpateen kulu-kulu Iko angga to ma'pasa' mairi' to ma'tammuan angga to ma'baluk-baluk

Rampananko balukammu annako pa'balilimmu pa'pasibasa-basammu
Fais attention au pas de la personne en transe veille aux pas des monte-au-ciel prends garde à ce qu'il n'y ait pas de dépassement

Puis tu prononces des paroles puis tu dis des mots qui ne ravissent pas des expressions déliées par ta langue

Prends des mesures contre celui qui t'envahit qui ne se soucie pas du faucon qui n'honore pas les oiseaux des bois

Toi qui viens sur ce marché ceux qui viennent sur ce lieu de rencontre tous les vendeurs

Lâche ton commerce abandonne ton négoce mets de côté tes affaires [...]

Les paroles évoquent aussi l'arrivée des divinités par le refrain sape-sape to balanda, "cueillette de l'étranger». Sape, "cueillir, retirer une feuille» rappelle l'origine mythique du rituel. Balanda désigne les Hollandais et, par extension, l'Etranger. Selon mon traducteur Y.M. Paranoan, sapé-sapé désignerait les ornements personnels dont les Hollandais se servaient pour s'embellir : lunettes, habits, chaussures, armes et surtout couvre-chefs (p. 98). Ces ornements auraient été vus par les Toraja vers 1670, lors de l'entrée de leurs voisins Bugis sur leurs terres. Les Toraja considéraient les Bugis comme des gens autoritaires, aimant gouverner, et possédant des armes. Le nom des divinités ne pouvant être prononcé, le chant emploie des substituts: Boné et Balanda seraient le travestissement, le déguisement du nom des divinités.

Sans ce chant masculin, les femmes ne peuvent se mettre à danser. Ce sont souvent les mêmes femmes qui entrent en transe (Hollan \& Wellengkamp 1994: 127). Comment accèdent-elles à un état second? L'impact du chant sur les danseuses tient à la puissance énergétique des voix, aux secousses, au rôle attribué au verbe. La danse provoque un essoufflement qui met certains participants dans un état émotionnel intense et qui les conduit à "faire divinité " (ma'deata). Le tempo de la profération ne varie pas, il n'y a pas d'accélération, ni aucune progression musicale. C'est davantage la prolongation de la répétition d'un simple, motif hurlé, ajoutée aux sauts qui provoque la "démence" des femmes.

Dans ce premier type, le "chant de la venue des divinités" déclenche une crise pour ceux qui l'entendent et non pour ceux qui l'exécutent. II est une incitation aux sauts qui provoquent un épuisement musculaire et une désorientation spatiale. 
Depuis quelques années, avec l'intensification de la christianisation, le nombre d'officiants rituels diminue. Quand il n'y a pas d'officiant pour mener le rituel, personne ne connaît les vers; le chant est alors hésitant et les femmes ne tombent pas en transe, ainsi, l'efficacité du rituel est remise en cause (Coville 1989: 122). Une fois les divinités descendues sur le champ cérémoniel, un second type de chant est exécuté.

\section{2. "Chant de l'être en divinité" (gelong kandeatan)}

Le second chant est chanté quand plusieurs femmes sont déjà délirantes. N'ayant pas pris part à la danse, un homme se dégage du groupe et commence par se flageller avec les feuilles de cordyline, boit de la boue, puis commence à se mutiler: il s'incise le front, la langue ou le ventre à l'aide d'un poignard (mantere). Pendant ce temps, un chant très doux et lent est exécuté par un chœur féminin, plus rarement mixte, communément appelé "chant des femmes» (gelong bainé). Cet homme applique ensuite ses mains sur un enfant. Etrangement, le sang qui commence à perler sur son front ou sur son ventre s'arrête rapidement si, disent-ils, le chant est bien exécuté.

Un peu plus loin et dans le même temps, une femme délirante s'agite en tous sens; derrière elle, un homme la contient à l'aide d'un couteau qu'il lui applique sur la taille. Une autre femme, hébétée, monte sur le tambour, puis redescend. D'autres personnes continuent à se flageller ou à s'appliquer la lame d'un couteau enroulée dans la feuille de cordyline sur leurs bras. La séance s'achève quand les personnes en transe reprennent conscience.

Ce second type de transe se distingue du premier par des actes "fakiristes" réservés à des hommes. C'est ici une transe de possession maîtrisée qui se solde par une démonstration d'invulnérabilité du corps au poignard. Le sang du guérisseur a pour fonction de guérir les malades:

Ke den to makuyu manuk sola to makuyu langkan

Mai maimoko inde'

Dipopedampi rara'na

dipotamba' kaisse'na

Make pabu'tumi mai

make baen-baenanmi

Ditadoi palakunna

diben lalan inaanna

Tungkalolong diara'na

lalan inayanna Puang
Si quelqu'un est malade continuellement et s'il est sans cesse grelottant

Viens alors ici

Son sang servira de remède le fruit rouge le guérira [...]

Allez, faites apparaître!

allez, donnez à voir!

Que sa volonté soit exaucée

que son désir soit satisfait

Il coule directement à sa poitrine

en accord avec la volonté des Dieux 
Pa'poraianna Deata

Tungka lolong diara'na

Napopedampi to tumbang

napotamba' to malangi'

inde to kandeatan
Tel le souhaitent les Divinités

II coule directement à sa poitrine

II guérit la personne en transe

il soigne le va-t-au ciel

voici les êtres en-divinité

Sur le plan musical, ce chant diffère totalement du premier type: le chœur, à l'unisson, se fait lancinant. La musique ne sert plus ici à déchaîner mais à apaiser. Les phrases sont simples, mélismatiques, l'ambitus restreint - il n'excède pas un ton -, le chant, homophone est composé de rythmes élémentaires:

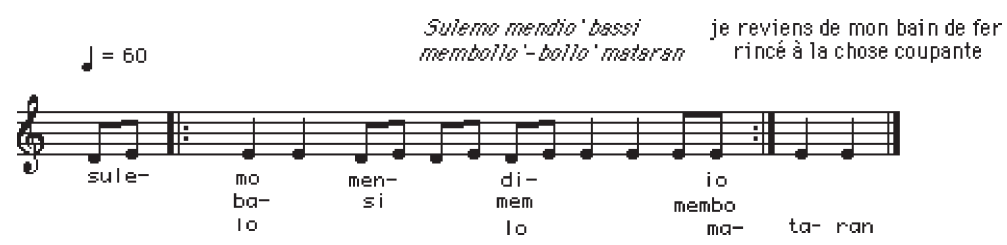

Ambitus restreint du chant de transe gelong bainé, CDM plage 24

Le plus souvent, l'ambitus ne dépasse pas un demi-ton:
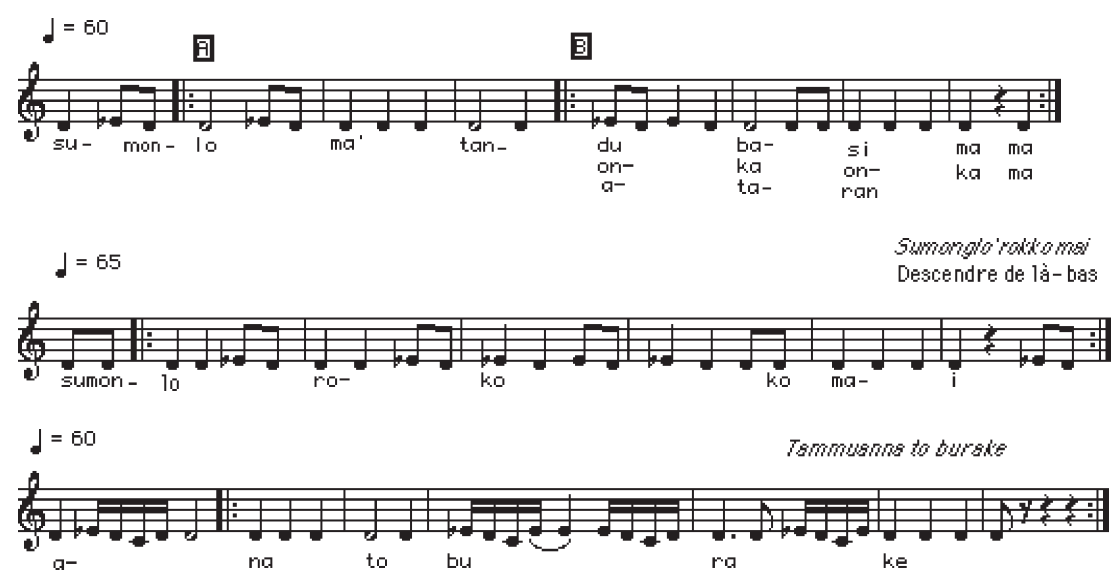

Que la plupart des motifs soient accentués tous les trois temps rappelle l'importance de la triade et du chiffre 3 dans ce rituel. Comment expliquer la récurrence de ces triades dans les manifestations visuelles (mâts tridents), poétiques (tercets), sonores (structures à trois temps), littéraires (triades de personnages) et rituelles (mélange des trois sangs d'animaux)? L'ethnologue Tsintjlonis (1997: 253) associe le chiffre 3 à la mobilité, au développement et à l'imminence, et le chiffre 4 à l'immobilité et à la fixité. S'agirait-il de l'imminence du divin, de la venue des divinités sur terre? 
Ce chant des femmes est appelé par différents noms: "chant de l'êtreen-divinité" (gelong kandeatan), "chant de guérison" (gelong ma'pakatana) ${ }^{19}$ ou "chant de celui qui se scarifie" (sampa-sampa to mantere). Lors du rituel, il n'est exécuté que par fragments. Les femmes choisissent des séquences dans un stock de vers ordonnés. Dans la version complète qui me fut communiquée par l'officiant Ne'Lumbaa, au début, le chant appelle les divinités à descendre sur le lieu cérémoniel:

$\begin{array}{ll}\begin{array}{l}\text { Sumonglo' ma' tanduk bassi } \\ \text { ma'ongka-ongka mataran } \\ \text { ma'patondon oda-oda }\end{array} & \begin{array}{l}\text { Descendre avec les cornes de fer } \\ \text { armé de la chose aiguisée } \\ \text { orné de l'arme tranchante }\end{array} \\ \begin{array}{l}\text { Oda-oda talaomo } \\ \text { sudidi tatiangka'mo } \\ \text { sumonglo' rokkoko moko }\end{array} & \begin{array}{l}\text { Arme tranchante, allez, en route! } \\ \text { chose coupante, en avant! } \\ \text { descendez ensemble ici }\end{array} \\ \begin{array}{l}\text { Laoko sambalin mai } \\ \text { bu'tu lamban diong mai }\end{array} & \text { Venez de par là-bas } \\ \text { traversez de l'autre rive }\end{array}$

Ce qui est convoqué, c'est l'arme tranchante, la divinité du fer qui servira à l'incision de la peau. L'importance de la vision est ici centrale. Une fois les divinités et l'arme tranchante arrivées, l'officiant s'incise la peau et le chœur chante. Pendant que le sang coule, il invite alors une personne à guérir par son sang:

19 De tana, matana, "content, satisfait; sain, guéri, sauf»; pakatana: "divertir, faire disparaître, consoler".

20 La traduction de ce vers est problématique. Lino signifie "monde». Popentolinomi peut signifier "faire apparaître" ou "rendre humain".
21 II s'agit de l'arrivée des divinités et esprits. Sesean est une des principales montagnes de Toraja où logeraient les divinités.

22 Lindo Tau, "Visage Humain", désigne la montagne Sesean; à son sommet se trouve une pierre similaire à un visage humain. 
Lolo tabang pedampinna

lassege' pepamurru'na

pi'tok peba'na-ba'nanna

Nasule kale datunna

tokuli' ampu lembangna

urruru tampa todolona

sola tasim bulayanna

Sulemo' mendio' bassi

membollo-bollo mataran

mellangi' sanda ura'na
La pointe de la cordyline est notre remède la pousse lassege' est notre traitement la plante pi'tok notre pharmacopée

Le corps revient au début avec une forme intacte il retrouve la forme du début avec une force rayonnante

De retour du bain de fer rincé à la chose coupante lavé à toutes sortes de couteaux ${ }^{23}$

Le bain de fer évoque l'épreuve du couteau que s'inflige l'homme qui se scarifie. La puissance dramatique est liée au décalage du sujet de l'énonciation: le chœur chante à la première personne du singulier le "drame" de celui qui se scarifie pour la collectivité.

Plus loin, le chant évoque le voyage de la personne-en-divinité dans le monde supérieur:

Dayo turunan ditoke'

dayo bubun dianginni

Turunan dibangke' rara'

rebadun rayu-rayu

dibala batu bulayan
Là-haut le puits est suspendu

là-haut la source pend dans le vent

Puits bordé de perles d'or

endigué de pierres précieuses

clôturé de pierres d'or

Si la personne demande un tambour, elle part alors en voyage par le biais du tambour. Le chœur chante la pensée de la personne en transe:

Sulemo mendio' bassi

membollo-bollo mataran

melangi' sanda ura'na

Tang piak tang kaumammo

tang tingkale talemammo

tangkan tipa'dua mammo
Je reviens de mon bain de fer

rincé à la chose coupante

lavé à toutes sortes de lames ${ }^{24}$

Pas seulement fendu comme le bois

pas seulement tranché en partage

pas seulement coupé en deux

Le tambour et le fer sont des adjuvants de la guérison. L'officiant Lumbaa insiste sur la nature magique du chant: "Certaines fois, le sang ne s'arrête pas, malgré les flagellations à l'aide des feuilles de cordyline. Cela arrive quand le chant est

23 En bugis, langi' désigne un shampoing de cendre de riz.

24 Ura': partie du corps en forme de fil, veine, artère, nerf, nervures, tendons, tuyaux. Autre traduction possible: "lavé à toutes sortes de choses aiguisées". 
Tableau 1. Comparaison des deux types de transe du rituel maro observé à Torea

\begin{tabular}{|c|c|c|}
\hline & Premier chant & Deuxième chant \\
\hline $\begin{array}{l}\text { fonction du } \\
\text { chant }\end{array}$ & $\begin{array}{l}\text { faire descendre les divinités sur le } \\
\text { lieu d'énonciation }\end{array}$ & $\begin{array}{l}\text { prophylactique ou curative } \\
\text { (soin par le sang, par le fer) }\end{array}$ \\
\hline $\begin{array}{l}\text { qui entre } \\
\text { en transe? }\end{array}$ & $\begin{array}{l}\text { des femmes. } \\
\text { Elles se flagellent, marchent sur } \\
\text { des braises, font des jeux déments. } \\
\text { Démence mimée }\end{array}$ & $\begin{array}{l}\text { un ou deux hommes se transpercent le } \\
\text { front, la langue ou le ventre, boivent de } \\
\text { la boue, appliquent leur sang sur des } \\
\text { enfants, les portent sur les épaules, fla- } \\
\text { gellent des personnes à l'aide de feuilles } \\
\text { Ils font office de guérisseurs tempo- } \\
\text { raires. } \\
\text { pas de crise de démence } \\
\text { actes fakiristes }\end{array}$ \\
\hline $\begin{array}{l}\text { retour à l'état } \\
\text { normal }\end{array}$ & $\begin{array}{l}\text { la personne en transe exige qu'on } \\
\text { lui apporte quelque chose, un } \\
\text { objet, un instrument de musique... }\end{array}$ & $\begin{array}{l}\text { le sang s'arrête de couler grâce au } \\
\text { chant }\end{array}$ \\
\hline $\begin{array}{l}\text { noms des } \\
\text { personnes } \\
\text { en transe }\end{array}$ & $\begin{array}{l}\text { to tumbang } \\
\text { to malangi" "va-t-au ciel» } \\
\text { to karondonan "celui qui fait } \\
\text { tomber" } \\
\text { to naala deata "pris par la divinité" }\end{array}$ & to mantere "celui qui se scarifie" \\
\hline volontaire & transe volontaire encadrée & transe volontaire maîtrisée \\
\hline \multicolumn{3}{|c|}{ MUSIQUE } \\
\hline nom du chant & $\begin{array}{l}\text { "chant de la venue des divinités" } \\
\text { gelong ma'pasae deata }\end{array}$ & $\begin{array}{l}\text { "chant de celui qui se scarifie" } \\
\text { sampa-sampa to mantere }\end{array}$ \\
\hline $\begin{array}{l}\text { nom commun } \\
\text { du chant }\end{array}$ & $\begin{array}{l}\text { "sauts des hommes" } \\
\text { nondo muane }\end{array}$ & $\begin{array}{l}\text { "chant des femmes" } \\
\text { gelong bainé }\end{array}$ \\
\hline contenu textuel & $\begin{array}{l}\text { arrivée des divinités sur le lieu } \\
\text { adresses au public }\end{array}$ & $\begin{array}{l}\text { description du voyage et du retour } \\
\text { description de la scarification et du } \\
\text { sang, de la guérison }\end{array}$ \\
\hline chanteurs & chœur d'hommes & $\begin{array}{l}\text { chœur de femmes } \\
\text { (+ quelques hommes) }\end{array}$ \\
\hline danseurs & $\begin{array}{l}\text { plusieurs femmes cheveux déliés } \\
\text { qui tournoient }\end{array}$ & absence de danse \\
\hline dynamique & chant saccadé, rythmé, vif, crié & chant lent, incantatoire \\
\hline \multicolumn{3}{|c|}{ MUSIQUE ET TRANSE } \\
\hline $\begin{array}{l}\text { fonction de } \\
\text { la musique }\end{array}$ & $\begin{array}{l}\text { la musique chantée par les } \\
\text { hommes accélère la crise des } \\
\text { femmes }\end{array}$ & $\begin{array}{l}\text { la musique chantée par les femmes } \\
\text { apaise le sang de l'homme }\end{array}$ \\
\hline type de transe & $\begin{array}{l}\text { inspiration (ou possession?) } \\
\text { femmes «musiquées» }\end{array}$ & $\begin{array}{l}\text { communion, voyage } \\
\text { officiant «musiqué» }\end{array}$ \\
\hline
\end{tabular}


faux; si le chant est juste, le sang s'arrête immédiatement et la blessure disparaît sans laisser de cicatrices" (officiant Lumbaa, 1993).

Contrairement au premier type de chant, celui-ci doit "calmer le jeu». En fait, tout comme le fer et la feuille de cordyline, il permet la guérison.

\section{Deux types de transe de possession}

Les deux types de chant correspondent à deux types de transe: le premier est une transe d'inspiration, induite - les femmes, "musiquées", sont investies par la divinité mais ne s'y identifient pas. Le second type, quant à lui, présente une étape intermédiaire entre la transe de possession et la transe chamanique. Dans le premier cas, les divinités descendent sur le lieu cérémoniel par l'intermédiaire des personnes en transe. Dans le second cas, les divinités descendent et, dans un mouvement inverse, les personnes en transe partent en voyage. Le guérisseur maîtrise son état et ne semble pas possédé. Pourtant, contrairement aux transes chamaniques, ce n'est pas sur le guérisseur que repose le poids principal du chant, mais sur le chœur des femmes, c'est-à-dire sur un groupe qui n'entre pas en transe. Ainsi, on ne peut parler véritablement dans ce cas ni de transe de possession, ni de transe chamanique. S'agirait-il de ce que Rouget nomme "transe de communion", une rencontre entre la divinité et le sujet, une forme de transe non identificatoire? Le tableau 1 récapitule les oppositions entre les deux chants.

La division des deux types de chant met en évidence, d'une part, deux transes différentes (cf. tableau) et, d'autre part, une nette division des rôles et des sexes. Le chant est conduit alternativement par les hommes puis par les femmes alors que les transes sont vécues de manière inverse par les femmes puis par les hommes. Que révèle cette inversion? Pourquoi, dans le mythe, la femme permetelle la guérison alors que le rituel présente un homme guérisseur?

En fait, ce n'est pas seulement un homme qui se charge de communiquer avec les divinités par le don de sang, mais également les femmes et les chanteurs, et en quelque sorte tous ceux qui prennent part à la fête et qui se dépensent en elle: tous sont les substituts de leur communauté. C'est à travers leur personne qu'est symboliquement transmis un capital collectif de substance énergétique. On comprend dès lors combien il est important que, dans un village, chaque famille participe au rituel, seul moyen d'échange collectif avec les divinités. Tous obtiennent la protection et la neutralité des divinités après leur avoir donné ce qui est le plus précieux. La contrepartie attendue de ce don se rapporte à l'existence: les divinités ne doivent pas empêcher la vie et sont tenues de ne provoquer ni maladie ni désordre. Ce qui est donné lors du maro doit valoir pour toute la période à venir, qui se prolongera sur le rituel bua' (rite suivant dans l'échelle sacrificielle).

Les chants et les récitations constituent la condition de possibilité de la relation entre les différents mondes (du haut, du bas, du milieu). Central dans le 
rituel, le chant initie ou inaugure des transformations: les divinités arrivent, les lames des couteaux tranchantes deviennent molles et inoffensives, les personnes en transe reviennent à la réalité, le sang s'arrête de couler. Le chant gelong, saccadé puis lénifiant, détermine deux types de transe: quand il est rapide, il fait entrer les femmes en divinité. Quand il est lent et lancinant, il laisse la place à des actes "fakiristes" supposés guérir les malades.

Je n'ai pas assez souligné l'importance des récitations psalmodiées par les prêtres rituels dans les maisons. Qu'il faille psalmodier un à un ces 14000 vers (précédent mythique) et non pas les prononcer à toute allure comme des mantras indique l'importance accordée au gelong maro. L'efficace générale repose donc sur l'ensemble des chants: récitations à l'intérieur et chants de transe à l'extérieur.

Comme dans tous les grands rituels toraja, la parole rituelle est l'élémentclé: c'est le chant qui appelle les divinités et c'est le chant qui les renvoie d'où elles étaient venues. Le rituel réussit grâce à la foi commune des participants qui croient dans le pouvoir des poignards, des feuilles de cordyline, des tissages et des mâts cérémoniels, des chants, des transes, et des tambours. Acte socio-cosmique, il participe au combat de la communauté contre un désordre provoqué par la maladie, réelle ou imaginée. En rétablissant l'ordre socio-cosmique, la société ancestrale est consolidée et se perpétue.

En raison de la christianisation, ce rituel maro, qui fait partie des rituels du soleil levant, a presque totalement disparu aujourd'hui. Néanmoins, un autre type de transe a vu le jour, par le biais du rite pentecôtiste dont les adeptes sont de plus en plus nombreux en pays toraja, devenu chrétien à $98 \%$. Mais les transes collectives se font à présent dans les églises pentecôtistes (pentekosta) au son de musiques importées et non plus sur les visions transmises par la poésie chantée du gelong maro.

\section{Références}

ATKINSON Jane Monnig

1989 The Art and Politics of Wana shamanship. Berkeley: University of California Press.

BECKER Judith

2000 "Listening Selves and Spirit Possession». The World of Music, Bamberg: OttoFriedrich University, XLII/2: 25-50.

2004 Deep listener: Music, Emotion and Transing. Bloomington: Indiana University Press.

BELO Jane

1960 Trance in Bali. New York: Columbia University Press

COVILLE Elisabeth

1988 A Single Word Brings to Life: the Maro Ritual in Tana Toraja (Indonesia). Ph. D., University of Chicago.

1989 "Centripetal Ritual in a Decentred World Changing Maro Performances in Tana Toraja». In Russell S. \& Cunningham C. (ed.): Changing lives, changing rites : ritual and 
social dynamics in Philippines and Indonesia uplands. Center for South and Southeast Asian Studies, University of Michigan: 103-131.

CRYSTAL Eric \& Shinji YAMASHITA

1987 "Power of Gods: Ma'Bugi' Ritual of the Sa'dan Toraja». In Rita Smith Kipp and Susan Rodgers (ed.): Indonesian Religions in Transition. Tucson" University of Arizona Press: 48-70.

FRIEDSON Steven

1996 Dancing Prophets: Musical Experience in Tumbuka healing. Chicago and London: University of Chicago Press.

JENSEN Gordon \& Luh Ketut SURYANI

1993 Transe and Possession in Bali: A Window on Western Multiple Personality, Possession Disorder, and Suicide. Oxford: Oxford University Press.

GOUK Penelope ed.

2000 Musical Healing in Cultural Contexts. London: Ashgate Press.

HOLLAN Douglas \& Jane WELLENKAMP

1994 Contentment and Suffering: Culture and Experience in Toraja. New York: Columbia University Press.

KARTOMI Margaret

1973 "Music and Trance in Central Java». Ethnomusicology XVII/2: 163-208.

NOOY-PALM Hetty

1979 The Sa'dan Toraja: A Study of Their Social Life and Religion (vol. I). The Hague: Martinus Nijhoff.

1986 The Sa'dan Toraja: A Study of their Social Life and Religion (vol. II). The Hague: Martinus Nijhoff.

RAPPOPORT Dana

1995 "Du repérage musical au travail de terrain ethnomusicologique en Indonésie Orientale». Cahiers de musiques traditionnelles 8: 13-32.

1995 Indonésie, Toraja, funérailles et fêtes de fécondité. 1 CD, collection CNRS-Musée de l'Homme, Le Chant du Monde, LDX 2741004.

1997 Musiques rituelles des Toraja Sa'dan: Musiques du Couchant, Musiques du Levant (Célèbes-Sud, Indonésie). Lille: Atelier National de Reproduction des Thèses, Sudoc.

1999 "Chanter sans être ensemble. Des musiques juxtaposées pour un public invisible». L'Homme 152: 143-162.

2004a "Ritual Music and Christianization in the Toraja's Highlands, Sulawesi». Ethnomusicology 48/4: 378-404.

2004b "Musique et morphologie rituelle chez les Toraja d'Indonésie». L'Homme 171172: 197-218.

à p. $\quad$ Chants de la terre aux trois sangs. Musiques du Couchant, musiques du Levant chez les Toraja Sa'dan de l'île de Sulawesi (Indonésie). + DVD-Rom, Paris: Epistèmes - Editions de la Maison des Sciences de l'Homme.

ROSEMAN Marina

$1991 \quad H e a l i n g$ Sounds from the Malaysian Rainforest, Temiar music and Medecine. Berkeley and Los Angeles: University of California Press.

ROUGET Gilbert

1990 [1980] La musique et la transe. Esquisse d'une théorie générale des relations de la musique et de la possession. Paris: Gallimard.

SIMON Artur

1987 Gendang Karo (North Sumatra): Trance and Dance of the Karo-Batak. 2 LP. Berlin: Museum Collection Berlin, ISBN 3886095134. 
TSINTJILONIS Dimitri

1999 "Being in Place and Being a Place. Sumanga' in Buntao». Bijdragen tot de Taal-, Landen Volkenkunde 155/4: 617-643.

2004 "The flow of life in Buntao'. Southeast Asian Animism reconsidered». Bijdragen tot de Taal-, Land-en Volkenkunde 160/4: 425-455.

2006 "Monsters and caricatures: spirit possession in Tana Toraja". Journal of the Royal Anthropological Institute 12: 551-567.

VEEN Henrik van der

1965 The Merok Feast of the Sa'dan Toraja. The Hague: Martinus Nijhoff.

1966 The Sa'dan Toradja Chant for the Deceased. The Hague: Martinus Nijhoff.

1979 Overleveringen en Zangen der Zuid-Toradja's. The Hague: Martinus Nijhoff.

VEEN Henrik van der \& J. TAMMU

1972 Kamus Toradja Indonesia. Rantepao: Jajasan Perguruan Kristen Toradja.

VOLKMAN Toby Alice

1988 "The Tree of Desire: a Toraja Ritual Poem». In James Fox (ed.): To Speak in Pairs: Essays on the Ritual Languages of Eastern Indonesia: 282-306. Cambridge: Cambridge University Press.

World of Music

1997 Numéro Spécial: "Music and healing in transcultural perspectives", Bamberg: OttoFriedrich University.

YAMADA Yoichi

1997 Songs of Spirits: an Ethnography of Sounds in a Papua New Guinea Society. Boroko (Port Moresby): Institute of Papua New Guinea Studies.

ZERNER Charles

1981 "Signs of the Spirits, Signature of the Smith: Iron forging in Tana Toraja». Indonesia $31: 89-112$.

RÉsumé Chez les Toraja de l'île de Sulawesi en Indonésie, les divinités sont convoquées par le biais de transes et de chants lors de certains rituels. L'article porte sur la place du chant dans l'un d'eux, le rituel maro, "fou», observé en 1993.

Durant six jours et six nuits, le rituel s'organise dans plusieurs maisons pour finir collectivement, le dernier jour, sur un grand champ cérémoniel, le "marché» entre humains et divinités. II doit permettre à la fois de soigner et de prévenir les maladies, de purifier le village et d'aider les âmes défuntes à remonter au Levant. Il implique l'ensemble de la collectivité locale. Les transes ont lieu les trois derniers jours dans les cours des maisons et sur le grand champ. De grands étendards tridents chargés de tissus ancestraux sont plantés.

L'étude des chants de transe dévoile une poésie signifiante, révélant des visions de corps, de chair, de sang, des visions colorées de voyage, de descente d'euphorie cérémonielle. D'autre part, la distinction de deux dynamiques musicales met à jour deux transes de nature différente. 